\title{
Lentivirus-mediated knockdown of MeCP2 inhibits the growth of colorectal cancer cells in vitro
}

\author{
NING SONG ${ }^{1 *}$, KEQIANG LI ${ }^{2 *}$, YAN WANG $^{1}$, ZONGYOU CHEN $^{1}$ and LIUBIN SHI ${ }^{1}$ \\ ${ }^{1}$ General Surgery Department, The Affiliated Huashan Hospital of Fudan University, Shanghai 200040; \\ ${ }^{2}$ Oncomolecular Biology Laboratory of Ningbo No. 2 Hospital, Ningbo, Zhejiang 315010, P.R. China
}

Received January 7, 2015; Accepted October 14, 2015

DOI: $10.3892 / \mathrm{mmr} .2015 .4612$

\begin{abstract}
Methyl-CpG-binding protein 2 (MeCP2) is a transcriptional repressor that has been implicated in tumor onset and progression. Compared with normal and other tumorous tissue, $\mathrm{MeCP} 2$ is highly expressed in well-differentiated adenocarcinoma and mucinous adenocarcinoma tissues, particularly at the invasion site of colorectal cancer tissues. The aim of the present study was to evaluate the potential of $\mathrm{MeCP} 2$ for use as a therapeutic target for human colorectal cancer. The DLD-1 colorectal cancer cell line was subjected to lentivirus-mediated short hairpin RNA-induced knockdown of MeCP2 and the effects on cell growth, cell cycle progression and cell migration were assessed. It was confirmed that lentivirus-mediated RNA interference successfully suppressed MeCP2 expression in vitro, which was demonstrated to result in reduced cell viability, cell cycle arrest in G0/G1 phase and inhibition of cell migration. These results indicated that $\mathrm{MeCP} 2$ may serve as a potential target for gene therapy of colorectal cancer.
\end{abstract}

\section{Introduction}

Colorectal cancer is a major cause of cancer-associated mortality worldwide (1). Although colorectal cancer can be effectively managed if detected early, the prognosis of patients with colorectal cancer is poor due to the recurrence and distant metastasis (2). It is therefore of high importance to identify proteins involved in colorectal tumorigenesis, which may be utilized as targets for therapeutic or diagnostic strategies.

Tumorigenesis is closely associated with genetic alterations and epigenetic modifications (3). Epigenetic modification results from promoter methylation and/or alterations in histone

Correspondence to: Dr Liubin Shi, General Surgery Department, The Affiliated Huashan Hospital of Fudan University, 12 Middle Wulumuqi Road, Jingan, Shanghai 200040, P.R. China

E-mail: liubinshi_80@126.com

*Contributed equally

Key words: cell cycle, cell migration, cell proliferation, colorectal cancer, methyl-CpG-binding protein 2, RNA interference modification in cancer cells (4). DNA methylation and histone modifications are interlinked via methyl-CpG-binding proteins (MeCPs), among which $\mathrm{MeCP} 2$ has an important role in establishing this interaction (5). MeCPs mediate transcriptional repression in a sequence-independent process involving the modification of the chromatin structure and histone acetylation levels $(6,7)$. Recent studies have shown that MeCP2 is involved in several human cancers types, including breast cancer (8), hepatocellular carcinoma (9), osteosarcoma (10) and endometrial cancer (11). Darwanto et al (12) revealed that $\mathrm{MeCP} 2$ was highly expressed in well-differentiated adenocarcinoma and mucinous adenocarcinoma tissues compared with normal and other tumorous tissues, particularly at the site of invasion of colorectal cancer tissues. The accumulation of a large number of $\mathrm{CpG}$ loci a at the 5'-flanking region of the $\mathrm{MeCP} 2$ gene suggests that epigenetic events may be involved in the regulation of the observed periodic plasticity of $\mathrm{MeCP} 2$ expression during cancer progression. Pancione et al (13) demonstrated in vivo as well as in vitro that $\mathrm{MeCP} 2$, upon its recruitment, causes transcriptional silencing of peroxisome proliferator-activated receptor $\gamma$ (PPARG) during colon tumorigenesis via exerting repressive effects on chromatin signatures, resulting in an increased cell-proliferative and invasive potential of colorectal cancer. To the best of our knowledge, the functional role of MeCP2 in the proliferation and migration of colorectal cancer cells has remained to be elucidated.

RNA interference (RNAi)-mediated gene silencing is a potential therapeutic strategy, which has been evaluated in clinical trials for a number of diseases $(14,15)$. Due to their minimal toxicity and ability of stable transgene expression, lentiviral vectors are among the most promising vehicles for efficient gene delivery in basic research as well as gene therapy $(16,17)$. The present study assessed the role of MeCP2 in colorectal cancer and silenced $\mathrm{MeCP} 2$ by lentivirus-mediated RNA interference in colorectal cancer cells to assess its effects on cell proliferation, the cell cycle and migration in vitro. The present study suggested that $\mathrm{MeCP} 2$ is a potential target for gene therapy in colorectal cancer.

\section{Materials and methods}

Cell lines and cell culture. The HCT116, DLD-1, SW480, LoVo, SW1116 and SW620 human colorectal cancer cell lines 
and the 293T human embryonic kidney cell line were obtained from the Cell Bank of the Chinese Academy of Science (Shanghai, China). HCT116, DLD-1, SW480 and LoVo cells were cultured in RPMI-1640 (Hyclone, Logan, UT, USA) containing $10 \%$ fetal calf serum (Biowest, Nuaillé, France). SW620 cells were cultured in L-15 medium (Sigma-Aldrich, St. Louis, MO, USA) containing 10\% fetal calf serum. SW1116 and 293T cells were cultured in DMEM (Hyclone) containing $10 \%$ fetal calf serum.

Reverse-transcription quantitative polymerase chain reaction $(R T-q P C R)$. The expression levels of MeCP2 in the DLD-1, HCT116, SW1116, SW620, SW480 and LoVo colorectal cancer cell lines were determined using RT-qPCR (CFX96; Bio-Rad Laboratories, Inc., Hercules, CA, USA). First, total RNA was extracted using TRIzol reagent (Invitrogen; Thermo Fisher Scientific, Waltham, MA, USA) and subjected to reverse transcription using Moloney murine leukemia virus reverse transcriptase (Promega Corp., Madison, WI, USA) according to the manufacturer's instructions. Subsequently $5 \mu \mathrm{l}$ of the resulting cDNA was amplified by PCR in a final volume of $20 \mu \mathrm{l}$ containing $0.8 \mu \mathrm{l}$ primers $(2.5 \mu \mathrm{M})$ and $10 \mu \mathrm{l}$ SYBR premix exTaq (Takara, Dalian, China). The thermocycling conditions were as follows: $95^{\circ} \mathrm{C}$ for $1 \mathrm{~min}$ followed by 40 cycles of $95^{\circ} \mathrm{C}$ for $5 \mathrm{sec}$ and $60^{\circ} \mathrm{C}$ for $20 \mathrm{sec}$, with the absorbance value read at the extension stage. Primers with the following sequences were used for RT-qPCR: MeCP2 forward, 5'-AGCAGTGAG AGCAGATGAGGTG-3' and reverse, 5'-GCCCAGGAT AGAGGAGACAAAGC-3'; $\beta$-actin forward, 5'-GTGGAC ATCCGCAAAGAC-3' and reverse, 5'-AAAGGGTGTAAC GCAACTA-3' (Genewiz, Inc., Suzhou, China). Data analysis was performed using the $2^{-\Delta \Delta C t}$ method (18). The assay was performed as three independent experiments.

Western blot analysis. To prepare protein extracts, DLD-1, HCT116, SW1116, SW620, SW480 and LoVo cells were scraped on the ice, collected by centrifugation $(12,000 \mathrm{x} \mathrm{g}$, $15 \mathrm{~min}, 4^{\circ} \mathrm{C}$ ) and incubated with freshly prepared $2 \mathrm{X}$ SDS lysis buffer [4\% SDS (Sangon Biotech Co., Ltd., Shanghai, China), $200 \mathrm{mM} \mathrm{NaCl}$ (Sangon Biotech Co., Ltd), 10\% glycerol (Sangon Biotech Co., Ltd.), 100 mM Tris (pH 6.8; Sangon Biotech Co., Ltd.) and 2 mM EDTA (Sangon Biotech Co., Ltd.)] for $10 \mathrm{~min}$. Following centrifugation, the protein concentration in the supernatant was determined using a BCA Protein Assay kit (Beyotime Institute of Biotechnology, Haimen, China). Equal amounts of protein (30 $\mu \mathrm{g}$ per experimental group) were boiled for $10 \mathrm{~min}$ in loading buffer [ $250 \mathrm{mM}$ Tris- $\mathrm{HCl}(\mathrm{pH} 6.8), 10 \%$ w/v SDS, 0.5\% w/v bromophenol blue (Sangon Biotech Co., Ltd.), $50 \%$ v/v glycerol, $5 \%$ w/v $\beta$-mercaptoethanol (Sangon Biotech Co., Ltd.)] prior to separation by $10 \%$ SDS-PAGE where samples were linearized at $80 \mathrm{~V}$ for $30 \mathrm{~min}$, then separated at $120 \mathrm{~V}$ for $90 \mathrm{~min}$. Subsequently, electrotransfer to polyvinylidene difluoride membranes (EMD Millipore, Billerica, MA, USA) was conducted at $300 \mathrm{~mA}$ for $1.5 \mathrm{~h}$. Membranes were blocked in Tris-buffered saline containing Tween 20 (TBST) with 5\% skimmed milk. Subsequently, the membranes were incubated with rabbit anti-human polyclonal MeCP2 (1:500; Proteintech Group, Inc., Chicago, IL, USA; cat. no. 10861-1-AP) and rabbit anti-human polyclonal GAPDH (1:3,000; Proteintech Group, Inc., Chicago, IL, USA; cat. no. 10494-1-AP) antibodies overnight at $4^{\circ} \mathrm{C}$, followed by incubation with the secondary goat anti-rabbit IgG antibody (1:5,000; Santa Cruz Biotechnology, Inc., Dallas, TX, USA; cat. no. sc-2054) for $2 \mathrm{~h}$ at room temperature. The membrane was washed three times with TBST prior to each step. Protein bands were visualized using the ECL prime ${ }^{\mathrm{TM}}$ blotting system (GE Healthcare, Little Chalfont, UK).

Lentiviral packaging vector. The lentiviral backbone plasmid pFH-L as well as the pHelper plasmids pVSVG-I and pCMV $\Delta$ R8.92 were purchased from Shanghai Hollybio Co. Ltd., (Shanghai, China). pFH-L containing RNA polymerase III promoter $\mathrm{H} 1$ initiates the expression of the inserted small hairpin (sh)RNA sequence, which continuously silences $\mathrm{MeCP} 2$ in the host cells. In addition, $\mathrm{pFH}-\mathrm{L}$ expresses the reporter gene green fluorescent protein GFP with activation via the cytomegalovirus promoter.

Construction of lentiviral vectors. Short-hairpin RNAs (shRNAs) specifically targeting human MeCP2 were designed based on the GenBank information for MeCP2 (ID, NM_004992.3). The shRNA targeting human MeCP2 (shMeCP2, 5'-GCCGTGAAGGAGTCTTCTATCCTCGAG GATAGAAGACTCCTTCACGGC-3') and the negative control shRNA (shCon, 5'-GCGGAGGGTTTGAAAGAA TATCTCGAGATATTCTTTCAAACCCTCCGCTTTTTT-3') were designed and synthesized by Shanghai Hollybio Co. Ltd. (Shanghai, China). Following annealing of the two oligos in annealing buffer [10 $\mathrm{mM}$ Tris (pH 8.0), $50 \mathrm{mM} \mathrm{NaCl}$ and $1 \mathrm{mM}$ EDTA] at $60^{\circ} \mathrm{C}$ for $20 \mathrm{sec}$, the resulting duplex DNAs were cloned into the lentiviral vector pFH-L (Shanghai Hollybio) and transfected into competent DH5 $\alpha$ E. coli cells (Tiangen Biotech Co., Ltd., Beijing, China). DNA sequencing was used to verify the positive clones.

For recombinant amplification of the vectors, 293T cells were co-transfected with MeCP2 RNAi lentiviral expression vector and control vector with the packaging vectors pVSVG-I and pCMVAR8.92 (Shanghai Hollybio) following the manufacturer's instructions for Lipofectamine 2000 (Invitrogen). Following $48 \mathrm{~h}$ of transfection, supernatants containing the lentiviruses $\mathrm{Lv}$-shCon or $\mathrm{Lv}$-shMeCP2 were harvested, which were purified using ultracentrifugation prior to determination of the lentiviral titer.

Lentiviral transfection. DLD-1 cells in the logarithmic growth phase were seeded into six-well plates at $5 \times 10^{4}$ per well and cultured overnight. The lentiviruses were transfected into the cells at a multiplicity of infection of 60. Successfully transfected cells were identified by detection of GFP using a fluorescent microscope (BX50; Olympus Corporation, Tokyo, Japan), with the percentage of GFP-positive cells representing the transfection efficiency. Cells were harvested at day four of transfection and the knockdown efficiency of MeCP2 was evaluated by RT-qPCR and western blot analysis.

Proliferation assay. DLD-1 cells were harvested at day four of transfection and seeded into a 96-well plate at a density of $3 \times 10^{3}$ cells/well in triplicate. Following incubation for 1, 2, 3, 4 or 5 days with the media replaced every other day, cells were subjected to the 3-[4,5-dimethylthiazol-2-yl]-2,5-diphenyl 


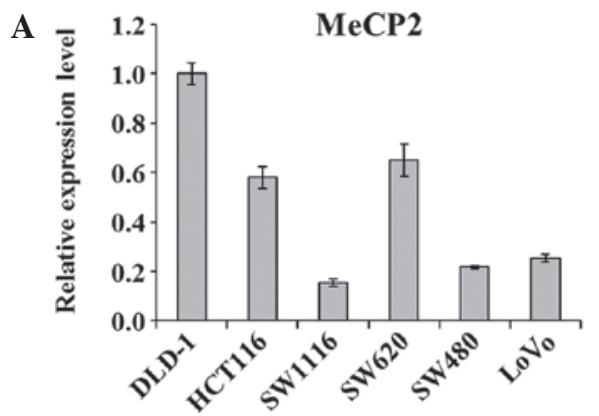

B

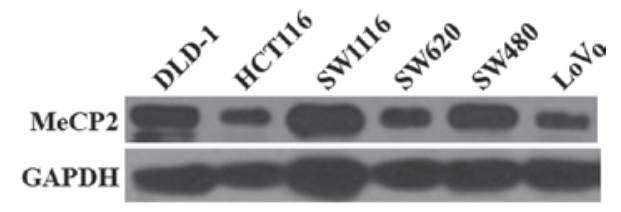

Figure 1. Expression levels of MeCP2 in human colorectal cancer cell lines. (A) Quantitative analysis of MeCP2 mRNA expression in DLD-1, HCT116, SW1116, SW620, SW480 and LoVo cell lines assessed by reverse-transcription quantitative polymerase chain reaction. $\beta$-actin was used as an internal control. (B) Representative western blots showing MeCP2 protein expression in the six cell lines. GAPDH protein was used as an internal control. Values are expressed as the mean \pm standard deviation $(\mathrm{n}=3)$. MeCP2, methyl-CpG-binding protein 2 .

tetrazolium bromide (MTT) assay. In brief, following addition of $20 \mu \mathrm{l}$ MTT solution ( $5 \mathrm{mg} / \mathrm{ml}$; Sigma-Aldrich) to each well, plates were incubated at $37^{\circ} \mathrm{C}$ for $4 \mathrm{~h}$. In order to dissolve the generated formazan crystals, wells were incubated with $100 \mu 1$ acidic isopropanol (10\% SDS, 5\% isopropanol and $0.01 \mathrm{~mol} / 1 \mathrm{HCl}$ ) at $37^{\circ} \mathrm{C}$ for $10 \mathrm{~min}$ with agitation. The absorbance of each well at $595 \mathrm{~nm}$ was determined using a microplate reader (Epoch; BioTek, Winooski, VT, USA). The assay was performed as three independent experiments.

Colony formation assay. DLD-1 cells were harvested at day four of transfection. Cells were seeded onto six-well plates at a density of 500 cells/well and allowed to form colonies over 11 days. Cell colonies were fixed in $4 \%$ paraformaldehyde and stained with crystal violet (Beyotime Institute of Biotechnology). Colonies ( $>50$ cells) were counted directly on the plate using a microscope. At least three independent experiments were performed to determine the number of colonies.

Cell cycle analysis. Following seven days of transfection, DLD-1 cells were harvested by trypsinization. Following suspension in phosphate-buffered saline (PBS), the cells were centrifuged $\left(1,000 \mathrm{x} \mathrm{g}, 5 \mathrm{~min}, 4^{\circ} \mathrm{C}\right)$ and fixed in $70 \%$ ethanol at $4^{\circ} \mathrm{C}$ for $1 \mathrm{~h}$. Following two washes with PBS, cells were re-suspended in $1 \mathrm{ml}$ PBS containing $500 \mathrm{U} / \mathrm{ml}$ RNase (Nanjing KeyGen Biotech. Co., Ltd., Nanjing, China) and incubated for $30 \mathrm{~min}$ at $37^{\circ} \mathrm{C}$. Subsequently, cells were incubated with $20 \mu \mathrm{g} / \mathrm{ml}$ propidium iodide (PI; Nanjing KeyGen Biotech. Co., Ltd.) for $30 \mathrm{~min}$ at room temperature in the dark to stain cellular DNA. Quantification of DNA was performed using a FACSCalibur flow cytometer (BD Biosciences, Franklin Lakes, NJ, USA) using CellQuest software, version 6.1 (BD Biosciences). A plot of the PI fluorescence signal at the FL2A peak vs. the cell count was used to discriminate G2/M cells from G0/G1 doublets. The relative populations of cells in $\mathrm{G} 0 / \mathrm{G} 1, \mathrm{~S}$ and $\mathrm{G} 2 / \mathrm{M}$ phases of the cell cycle were determined.

Migration assays. DLD-1 cells and Lv-shMeCP2-transfected DLD-1 cells ( $1 \times 10^{5}$ cells/well) in $200 \mu \mathrm{l}$ FBS-free RPMI 1640 medium were seeded into the upper chambers of Transwell plates (pore size, $8 \mu \mathrm{m}$ ), while the lower chamber was filled with $800 \mu 1$ RPMI 1640 containing 10\% FBS. After $24 \mathrm{~h}$ of incubation, cells on the upper surface of the membrane were removed using a cotton swab, and cells which had migrated to the lower side of the membrane were fixed with $10 \%$ methanol, stained with crystal violet and examined under a microscope. A total of five random high-power microscopic fields (magnification, $40 \mathrm{x}$ ) per filter were captured and the number of migrated cells was directly counted. Subsequently, the crystal violet on the lower membrane was dissolved in 33\% acetic acid and the absorbance of the eluant at $570 \mathrm{~nm}$ was determined using the microplate reader (Epoch). Three independent experiments were performed to determine the number of migrated cells.

Statistical analysis. SPSS software (version 16.0; SPSS, Inc., Chicago, IL, USA) was used for statistical analysis. All experiments were performed in triplicate and values are expressed as the mean \pm standard deviation where applicable. Statistically significant differences between groups were determined by Student's $t$-test. $\mathrm{P}<0.05$ was considered to indicate a statistically significant difference between values.

\section{Results}

MeCP2 expression in colorectal cancer cell lines. To explore the role of $\mathrm{MeCP} 2$ in human colorectal cancer, the expression levels of MeCP2 in six human colorectal cancer cells lines, DLD-1, HCT116, SW1116, SW620, SW480 and LoVo, were determined. As depicted in Fig. 1A, RT-qPCR analysis revealed that MeCP2 mRNA was expressed in all six colorectal cancer cell lines, with the highest expression in DLD-1 cells. Furthermore, western blot analysis revealed the presence of $\mathrm{MeCP} 2$ protein in colorectal cancer cells (Fig. 1B). Therefore, DLD-1 cells were selected for use in the subsequent experiments.

Lv-shMeCP2 efficiently mediates MeCP2 knockdown. To determine the transfection efficiency of Lv-shMeCP2 and Lv-shCon in DLD-1 cells, GFP expression was observed under a fluorescent microscope at $96 \mathrm{~h}$ after transfection. As shown in Fig. 2A, $>80 \%$ DLD-1 cells were GFP-positive in the shCon and shMeCP2 groups, indicating that Lv-shMeCP2and Lv-shCon were successfully constructed and that their transfection efficiency was high. Next, RT-qPCR and western blot analyses were performed to determine the mRNA and protein levels of MeCP2 in the shCon and shMeCP2 groups. As shown in Fig. 2B, Lv-shMeCP2 reduced the expression of 
A

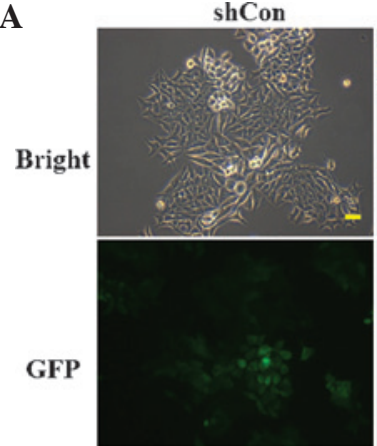

shMeCP

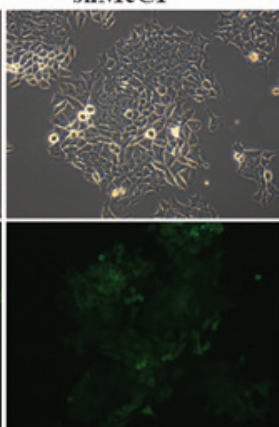

B

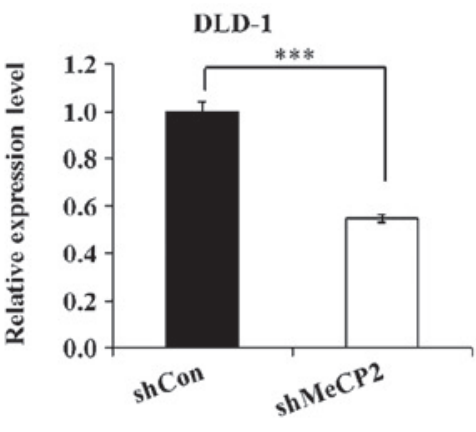

C

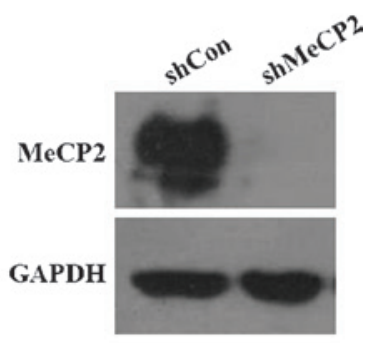

Figure 2. Efficient suppression of MeCP2 by lentivirus-mediated RNAi in colorectal cancer cells. (A) Fluorescence microscopy examination of the transfection efficiencies of lentivirus vector in DLD-1 cells (scale bar, $10 \mu \mathrm{m}$ ). (B) reverse-transcription quantitative polymerase chain reaction analysis of the knockdown of MeCP2 by lentivirus-mediated RNAi in DLD-1 cells. Values are expressed as the mean \pm standard deviation $(\mathrm{n}=3)$. **** $\mathrm{P}<0.001$. (C) Western blot analysis of the knockdown of MeCP2 by lentivirus-mediated RNAi in DLD-1 cells. Groups: shMeCP2, lentivirus-mediated MeCP2 RNAi group; shCon, lentivirus-mediated negative control RNAi group. RNAi, RNA interference; sh; small hairpin; MeCP2, methyl-CpG-binding protein 2.

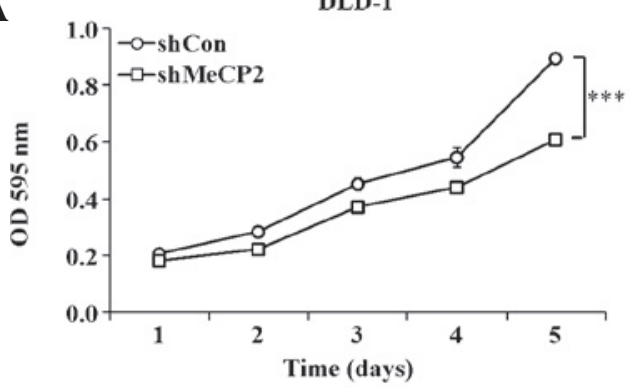

C

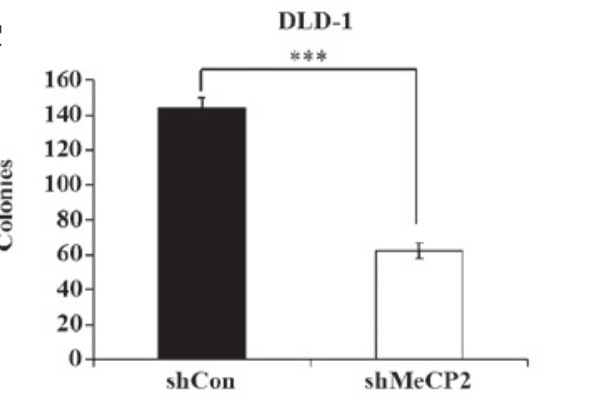

B

Crystal violet

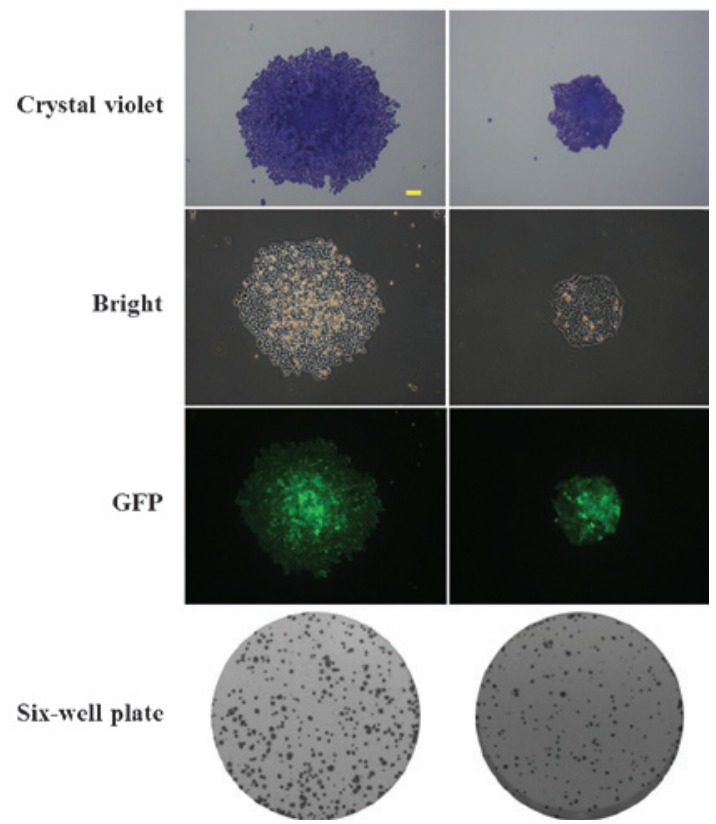

shMeCP2

Figure 3. MeCP2 depletion inhibits DLD-1-cell proliferation and colony formation. (A) Proliferation curves of shMeCP2 cells and shCon cells. (B) Representative images of cell colonies in the colony formation assay (scale bar, $25 \mu \mathrm{m}$ ). (C) Number of colonies of shMeCP2 and shCon cells. Values are expressed as the mean \pm standard deviation $(\mathrm{n}=3) .{ }^{* * *} \mathrm{P}<0.001$. Magnification, $\mathrm{x} 40$. OD, optical density; GFP, green fluorescence protocol; shCon, group transfected with control small hairpin RNA; shMeCP2, group transfected with small hairpin RNA targeting MeCP2; MeCP2, methyl-CpG-binding protein 2.

MeCP2 mRNA by $\sim 45.2 \%$ in DLD-1 cells relative to that in the shCon group $(\mathrm{P}<0.001)$. Western blot analysis validated that MeCP2 expression in DLD-1 cells relative to GAPDH was knocked down by transfection with shMeCP2, while that in the shCon group was still present (Fig. 2C). These analyses demonstrated that Lv-shMeCP2 efficiently knocked down MeCP2, which was therefore used in the subsequent experiments.

Knockdown of MeCP2 inhibits colorectal cancer cell growth. The effects of MeCP2 knockdown on the growth of colorectal cancer cells in vitro were assessed using MTT and colony formation assays. The cell proliferation was assessed using an MTT assay once daily over five days. As shown in Fig. 3A, MeCP2 silencing inhibited DLD-1 cell proliferation in a time-dependent manner. Compared with that in the shCon group, the cell viability in the shMeCP2 group was significantly reduced on days four and five $(\mathrm{P}<0.001)$. Furthermore, the colony formation capacity of DLD-1 cells transfected with Lv-shMeCP2 and Lv-shCon was investigated. As shown in Fig. 3B, the size of single colonies was observed using light and fluorescence microscopy. The sizes as well as the number of single colonies in the shMeCP2 group were reduced compared with those in the shCon group. As shown in Fig. 3C, the number of DLD-1 cell colonies in the shMeCP2 was significantly reduced to $62 \pm 5$, as compared with $144 \pm 6$ in the shCon group $(\mathrm{P}<0.001)$. Collectively, these results demonstrated that knockdown of MeCP2 by lentivirus-mediated siRNA inhibited the growth of colorectal cancer cells. 

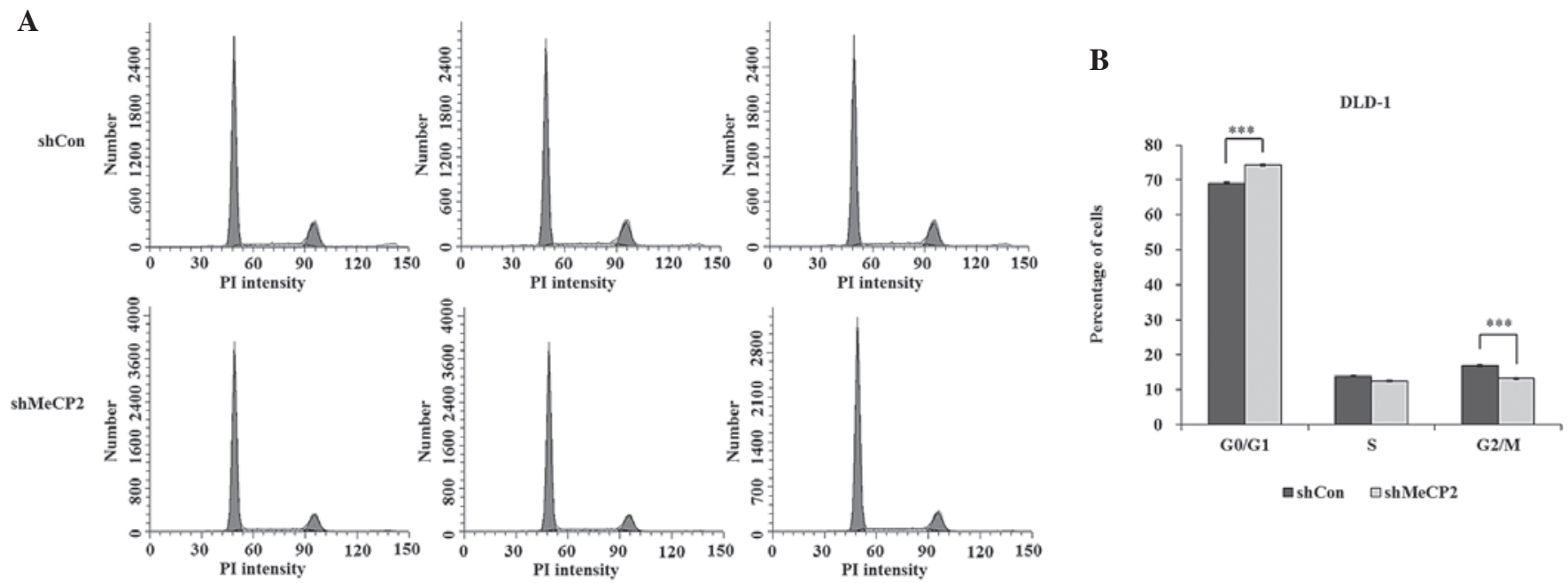

Figure 4. MeCP2 depletion affects cell cycle progression. (A) Flow cytometric analysis of cell cycle progression in the shCon and shMeCP2 groups. (B) Percentages of shMeCP2 and shCon cells in various phases of the cell cycle. shMeCP2 cells displayed a significantly elevated G0/G1 phase population and a decreased G2/M phase population compared with shCon cells. Values are expressed as the mean \pm standard deviation $(\mathrm{n}=3) .{ }^{* * * *} \mathrm{P}<0.001$. shCon, group transfected with control small hairpin RNA; shMeCP2, group transfected with small hairpin RNA targeting MeCP2; MeCP2, methyl-CpG-binding protein 2.

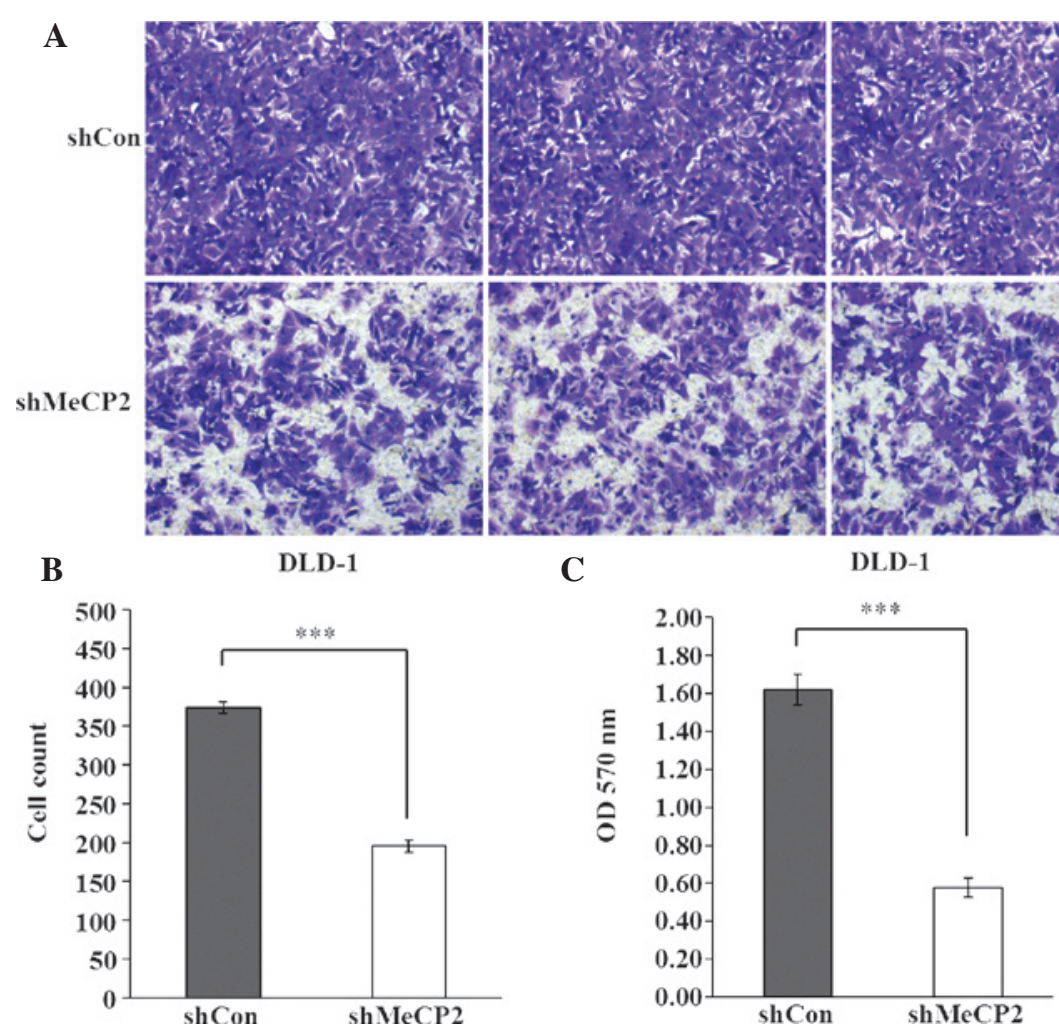

Figure 5. MeCP2 depletion inhibits DLD-1-cell migration. (A) Representative images of migrated cells stained with crystal violet on the lower side of the membrane in the migration assay. (B) Quantified numbers of migrated cells in the migration assay. (C) OD value of migrated cells. Values are expressed as the mean \pm standard deviation $(\mathrm{n}=3)$. ${ }^{* * * *} \mathrm{P}<0.001$. Magnification, $\mathrm{x} 40$. shCon, group transfected with control small hairpin RNA; shMeCP2, group transfected with small hairpin RNA targeting MeCP2; MeCP2, methyl-CpG-binding protein 2; OD, optical density.

Knockdown of MeCP2 causes cell-cycle arrest in G0/G1 phase in DLD-1 cells. To evaluate the effects of MeCP2 knockdown on the cell cycle distribution, PI staining followed by flow cytometric analysis was performed. The proportion of cells in G0/G1 phase was $74.19 \pm 0.31$ and $69.12 \pm 0.18 \%$ in the shMeCP2 and shCon groups, respectively, while the proportion of cells in $S$ phase was $12.54 \pm 0.21$ and $13.92 \pm 0.14 \%$, G2/M-phase was $13.28 \pm 0.29$ and $16.96 \pm 0.31 \%$, respectively.
$\mathrm{MeCP} 2$ silencing resulted in a statistically significant increase in the $\mathrm{G} 0 / \mathrm{G} 1$-phase population $(\mathrm{P}<0.001)$ as shown in Fig. 4A and B. These results indicated that downregulation of $\mathrm{MeCP} 2$ expression caused cell-cycle arrest in G0/G1 phase.

Knockdown of MeCP2 reduces DLD-1 cell migration. In order to explore the effects of MeCP2 knockdown on DLD-1 cell migration, a Transwell migration assay was performed. 
As shown in Fig. 5A-C a large proportion of cells in the shCon group migrated to the lower surface of the filter, while the number of migrated cells in the shMeCP2 group was significantly decreased by $47.6 \%(\mathrm{P}<0.001)$. These results indicated that downregulation of $\mathrm{MeCP} 2$ inhibited the migratory ability of colorectal cancer cells.

\section{Discussion}

$\mathrm{MeCP} 2$ has been detected in the majority of colorectal cancer tissues, particularly at the invasion site of cancers (12). To explore the association between MeCP2 and colorectal tumorigenesis, the expression of MeCP2 in colorectal cancer cells was inhibited through lentivirus-mediated RNAi. The results of the MTT assay performed in the present study indicated that downregulation of MeCP2 expression inhibited cell proliferation. In line with this finding, the colony formation assay showed that knockdown of MeCP2 inhibited the colony formation ability of colorectal cancer cells. These findings indicated that MeCP2 promotes cancer cell growth and suggested that $\mathrm{MeCP} 2$ may have an important role in the early stage of colon tumor development. To explore the potential underlying mechanism the role of $\mathrm{MeCP} 2$ in the growth of colorectal cancer cells, DLD-1 cells with stable knockdown of MeCP2 were subjected to flow cytometric cell cycling analysis. It was found that the specific downregulation of MeCP2 in DLD-1 cells led to G0/G1-phase arrest. It is known that in G0/G1 phase, cells do not divide, increase in size and accumulate nutrients (19). In order to proliferate, cells must enter $\mathrm{S}$ phase for DNA synthesis, while blocking of the G1/S-phase transition inhibits proliferation. MeCP2 acts as a transcriptional repressor to control gene expression in mammalian cells, which it exerts through non-specific binding to methylated $\mathrm{CpG}$ islands (20), thereby preventing DNA binding of transcription factors such as Sp1, which results in histone deacetylase-mediated alteration of the chromatin structure (21). Furthermore, it has been revealed that upon its overexpression, $\mathrm{MeCP} 2$ regulates E-cadherin (E-cad) expression in colorectal cancer; with ongoing tumor progression, loss of E-cad expression was shown to lead to the de-differentiation of human carcinomas in vitro and in vivo (12). Due to the critical function of MeCP2 on E-cad expression, it can be hypothesized that the observed reduction of cell proliferation following MeCP2 knockdown may be due to the inhibition of de-differentiation through loss of E-cad suppression.

Colorectal cancer is a highly metastatic malignancy. However, the impact of MeCP2 on colorectal cancer-cell migration has not been studied in detail. Therefore, the present study examined the effects of MeCP2 knockdown on the migration of DLD-1 cells using a Transwell migration assay. The results demonstrated that downregulation of MeCP2 expression in DLD-1 cells markedly reduced their migration capacity in vitro. These results corresponded to a recent study reporting that $\mathrm{MeCP} 2$ silencing inhibited osteosarcoma cell proliferation, migration and invasion (10). The E-cad gene encodes a cell-surface adhesion protein that has a crucial role in homotypic cell-cell adhesion and maintenance of epithelial morphology, while loss of E-cad expression and function inhibits cell-cell adhesion, thereby inducing tumor-cell invasion and metastasis (12). Therefore, MeCP2 depletion in the present study was likely to have inhibited the loss of E-cad expression, which led to the observed reduction of colorectal cancer-cell migration.

Although the underlying molecular mechanisms of the effects of MeCP2 gene silencing have not been clearly demonstrated, the present study evidenced that MeCP2 knockdown was capable of inhibiting colorectal cancer cell proliferation and migration. Thus, it is speculated that MeCP2 may be a molecular therapeutic target in the treatment of colorectal cancer.

In conclusion, the present study demonstrated that lentivirus-mediated RNAi with MeCP2 expression significantly inhibited colorectal cancer cell proliferation and migration. Therefore, MeCP2 represents a novel molecular target for the treatment of colorectal cancer.

\section{Acknowledgements}

The authors are grateful for the financial support from the Natural Science Foundation of Shanghai (no. 12ZR1404200).

\section{References}

1. Wang WS, Chen PM and Su Y: Colorectal carcinoma: From tumorigenesis to treatment. Cell Mol Life Sci 63: 663-671, 2006.

2. Li Z, Tian T, Hu X, Zhang X, Li L, Nan F, Chang Y, Wang X, Sun Z, Lv F and Zhang M: Targeting Six 1 by lentivirus-mediated RNA interference inhibits colorectal cancer cell growth and invasion. Int J Clin Exp Pathol 7: 631-639, 2014

3. Jones PA and Baylin SB: The epigenomics of cancer. Cell 128: 683-692, 2007.

4. Esteller M: Epigenetics in cancer. N Engl J Med 358: 1148-1159, 2008.

5. Hite KC, Adams VH and Hansen JC: Recent advances in MeCP2 structure and function. Biochem Cell Biol 87: 219-227, 2009.

6. Bestor TH: Gene silencing. Methylation meets acetylation. Nature 393: 311-312, 1998.

7. Nan X, Ng HH, Johnson CA, Laherty CD, Turner BM, Eisenman RN and Bird A: Transcriptional repression by the methyl-CpG-binding protein MeCP2 involves a histone deacetylase complex. Nature 393: 386-389, 1998.

8. Ray BK, Dhar S, Henry C, Rich A and Ray A: Epigenetic regulation by Z-DNA silencer function controls cancer-associated ADAM-12 expression in breast cancer: Cross-talk between MeCP2 and NF1 transcription factor family. Cancer Res 73: 736-744, 2013.

9. Zhao LY, Zhang J, Guo B, Yang J, Han J, Zhao XG, Wang XF, Liu LY, Li ZF, Song TS and Huang C: MECP2 promotes cell proliferation by activating ERK1/2 and inhibiting p38 activity in human hepatocellular carcinoma HEPG2 cells. Cell Mol Biol (Noisy-le-grand) Suppl 59: OL1876-OL1881, 2013.

10. Meng G, Lv Y, Dai H, Zhang X and Guo QN: Epigenetic silencing of methyl-CpG-binding protein 2 gene affects proliferation, invasion, migration and apoptosis of human osteosarcoma cells. Tumour Biol 35: 11819-11827, 2014.

11. Chu Y, Wang Y, Zhang G, Chen H, Dowdy SC, Xiong Y, Liu F, Zhang R, Li J and Jiang SW: Chromatin composition alterations and the critical role of $\mathrm{MeCP} 2$ for epigenetic silencing of progesterone receptor-B gene in endometrial cancers. Cell Mol Life Sci 71: 3393-3408, 2014

12. Darwanto A, Kitazawa R, Maeda S and Kitazawa S: MeCP2 and promoter methylation cooperatively regulate E-cadherin gene expression in colorectal carcinoma. Cancer Sci 94: 442-447, 2003.

13. Pancione M, Sabatino L, Fucci A, Carafa V, Nebbioso A, Forte N, Febbraro A, Parente D, Ambrosino C, Normanno N, et al: Epigenetic silencing of peroxisome proliferator-activated receptor $\gamma$ is a biomarker for colorectal cancer progression and adverse patients' outcome. PLoS One 5: e14229, 2010.

14. Lin HC, Wu CL, Chen YL, Huang JS, Wong TY and Yuan K: High-level $\beta 1$-integrin expression in a subpopulation of highly tumorigenic oral cancer cells. Clin Oral Investig 18: 1277-1284, 2014. 
15. Ren W, Wang X, Gao L, Li S, Yan X, Zhang J, Huang C, Zhang Y and Zhi K: miR-21 modulates chemosensitivity of tongue squamous cell carcinoma cells to cisplatin by targeting PDCD4. Mol Cell Biochem 390: 253-262, 2014.

16. Yu LL, Chang K, Lu LS, Zhao D, Han J, Zheng YR, Yan YH, Yi P, Guo JX, Zhou YG, et al: Lentivirus-mediated RNA interference targeting the $\mathrm{H} 19$ gene inhibits cell proliferation and apoptosis in human choriocarcinoma cell line JAR. BMC Cell Biol 14: 26, 2013.

17. Sun W, Yao L, Jiang B, Guo L and Wang Q: Spindle and kinetochore-associated protein 1 is overexpressed in gastric cancer and modulates cell growth. Mol Cell Biochem 391: 167-174, 2014.

18. Pfaffl MW, Horgan GW and Dempfle L: Relative expression software tool $\left(\mathrm{REST}^{\circ}\right)$ for group-wise comparison and statistical analysis of relative expression results in real-time PCR. Nucleic Acids Res 30: e36, 2002.
19. Zhang S, Yang X, Shi H, Li M, Xue Q, Ren H, Yao L, Chen X, Zhang J and Wang H: Overexpression of leucine aminopeptidase 3 contributes to malignant development of human esophageal squamous cell carcinoma. J Mol Histol 45: 283-292, 2014.

20. Nan X, Campoy FJ and Bird A: MeCP2 is a transcriptional repressor with abundant binding sites in genomic chromatin. Cell 88: 471-481, 1997.

21. Kudo S: Methyl-CpG-binding protein MeCP2 represses Spl-activated transcription of the human leukosialin gene when the promoter is methylated. Mol Cell Biol 18: 5492-5499, 1998. 